\title{
Disambiguation by Prioritized Circumscription
}

\author{
Ken Satoh \\ Hokkaido University \\ N13W8 Kita-ku Sapporo 060 Japan \\ ksatoh@db.huee.hokudai.ac.jp
}

\begin{abstract}
This paper presents a nethod of resolving anbiguity by nsing a variant of circuntscription, prortized circumseription. In a disambiguation tiask, human seems to use various preferences which latve varions strength. In prioritized cir (antscription, we can express these prefcrences as defeasible constratints with various strength and we infer the most preferable logical models which salisfy stronger constintints as much as possible. This representation is very natur. ral for disambiguation since we can re gard a logical interpretalion as a possible reading atd the most preferable logical models as the most preferable readings. We argue that prioritized circum scription is another promising method for the task. We also discuss an inplementation of prioriti\%ed circunserip tion by a hicrarchical logic programming (HCI,P) lamguage.
\end{abstract}

\section{Introduction}

This papes presentis a method of disambignation task by a variant of circumscription, prioritized circumscription (McCarthy, 1986; Lifschiliz, 1985) and discuss its implementation by a lierarchical constraint logic programming (TICLP) language such as (Borning et al., 1989).

Disambigualion is a very important task in natural language processing. To resolve ambiguity, humans seem to use not only syntactic constraints but also varions levels of heuristics such as grammatical preferences (Hobbs, 1990) and semantic preferencess (Wilks, 1975).

For example, suppose that we have the following sentences.

John just saw a man with a telescope. (a)

He bought the telescope yesterday.

Although there is an ambiguity on monning of the phrase, "with a relescope" (the telescope is either used by John or carried by the man), we might conchude the prefered reading as follows.

From the above sentences, "He" would be equal to John because the subject tends to be continued to the next sentence and John probably had a telescope at the time of seeing a man from the sentence (b) and inertia of possession. Therefore, from this preferred reading, we conclude that the telescope is used as a device to see a man.

However, this reading is not final since at least the following preferences are involved in the abovo reading and these preferences can be defeated by stronger information.

Syntactic preference: The subject tends to be sontinumed.

Semantic preference: If a person buys something at time $i$, then he should have it at time $j$ where $i<j$.

In order to demonstrate defeasibility of profercnce rules, suppose the following sentence is added aftice the above sentences (a) and (b).

But, he gave the man the telescope (c) this morning.

Then, we might change a preferred reading that the man shonld have had a telescope and therefore, the telescope was carried by the man at the tinne of John's seeing the man. In this reading. at least, the following preference rule of another inertia of possession is used.

If a person gives something to the other person at time $i$, then the other person should have it at time $j$ where $i<j$.

This conflicts with the former semantic preference of incrtia of possession by buying, but the above preference is stronger than the former since the time of giving is later than the time of buying. Thtts, the former preference becomes no loner applicable by the new sentence.

This kind of revision of reating cannot be represented by inference in classical logic since in classical logic, once wo get a inferred result, we can no longer retract the result (monotonic property). 'l'herefore, to understand the: phenomena, we need other reasoning methods and in fact 
many researches have been using general reasoning fanmeworks in Artificial Intelligence such as abduction (Hobbs et al.. 1993), probabilistic network (Charniak and Goldman, 1989), truth maintenance system (Zernik and Brown, 1988), default logic (Quant: 1993) and conditional logic: (Lascarides, 1993). In this paper, we propose another alternative, that is, circumscription (McCarthy, 1986: Lifschit\%, 1985). Even though circumscription is one of the most popular formalisms in the conmmnity of nommonotonic reasoning research, it is surprising that very fow has examined feasibility of circumscription for disambiguation. O11r work of disambiguation by interpretation ordering is originated from (Satoh, 1991) and in a more recent work, Kameyama (Kameyama, 1994) has independently proposed usage of circunuscriplion for intierpretation of pronominal andphora.

In this paper, we explore this direction further. In circumscription, we give a preforence order over logical interpretations and consider the most preferable models. This representation nati1rally corresponds with a disambiguation task since we can regard a logical interpretation as a possible reading, and disambignation as a lask to get the most preferable reading among possible readings. Among variants of circumseription, prioritized circumseription is suitable to represent various strength of preference rules. In prioritized circumseription, we can divide preference rules into hierarchy and this hierarchy gives a priority relation over preferences. Therefore, we directly represent rules in the hierarelyy in prioritized circumscription.

We believe that circumscription has the following alvantages in the task of resolving ambiguity.

- Since we use a tirst-order predicate calculus for a basic langnage, we can represent varions kinds of information such as grammatical rules and semantical rules in one framework.

- There is ouly one extra nuderlying mechanism besides inference rules for the first-order predicate calcululs. that is, introducing an order over logical interpretations. Therefore, reasoning process can be understood casily compared to other mochanism using numerical reasoning or complex inference rules.

- We do not need to assign detiailed mumerical valnes to preference rules in order to express priority over preference rules. but just specify a preference level of the rules. This representation can be re garded as an assignment of qualitative strength for preference rules and reduces a burclen of representing a priority over preference rules greatily. Moreover, this prioritization is general since we cun represent a various kind of priority besides specilicity.

- It is important to retain possible readings if we can not. resolve ambiguity yet. In circumscription, wo can consider multiple preferable models, not necessary the single preferable model. So, if there are yet multiple possible readings as a result of disambiguation, we can keep these possible readings as multiple preferable morlels.

In this paper, we also discuss an implementation by using hierarchical constraint logic programming (HCLP) language such ass (Borning of al., 1989). HCLP language is similar to constraint logic programmiug language except that we can represent a constraint hierarchy. Thus, there is a correspondence between a solution of an HCLP language and the most preferable models of prioritized circumscription. In this paper, we use our HCLP language based on a boolean constraint solver to get the most preferable models from preference rules represented as boolean constraints in the HCLP' language. We demonstrate how the above example of the disambiguation is treated in the HCLP language.

\section{Prioritized Circumscription}

In this section, we briefly review prioritized circumscription. For sinplicity sake, we molify the defintion of prioritized circumscription by (McCarthy, 1986; Lifschitz, 1985). The difference is that we let all predicates vary and maximize preference rules whereas Lifschitz minimize abnormal predicates for preference rules.

Let, $\Phi(\mathbf{x})$ and $\Psi(\mathbf{x})$ be formulas with the same number of free variables $\mathbf{x}$. We say that $\Phi$ and $\Psi$ are similar. $\Phi \geq \Psi$ stands for $\forall \mathbf{x}(\Psi(\mathbf{x}) \supset \Phi(\mathbf{x}))$. We extend this notation to tuples of formulas $\Phi, \Psi$ where $\Phi=\Phi_{1}, \ldots, \Phi_{n}$ and $\Psi=\Psi_{1}, \ldots, \Psi_{n}$ and $\Phi$ and $\Psi$ are similar (each $\Phi_{j}$ and $\Psi_{j}$ are similar): $\Phi \geq \Psi$ stauds for $\wedge_{j=1}^{n} \Phi_{j} \geq \Psi_{j}$. Wo also write $\Phi \geq \Psi \wedge \Psi \geq \Phi$ as $\Phi=\Psi$ and $\Phi \geq \Psi \wedge \neg(\Psi \geq \Phi)$ as $\Phi>\Psi$.

Let a tuple of formulas $\Phi$ be broken into disjoint. partis $\Phi^{1}, \Phi^{2}, \ldots, \Phi^{k}$. Let $\Psi^{i}$ be similar to $\Phi^{i}$. We define $\Phi \succeq \Psi \stackrel{\text { def }}{=} \wedge_{i=1}^{k}\left(\wedge_{j=1}^{i \cdots 1} \Phi^{j}=\Psi^{j} \supset \Phi^{i} \geq \Psi^{i}\right)$. Wo also write $\Phi \succeq \Psi \wedge \neg(\Psi \succeq \Phi)$ as $\Phi \succ \Psi$.

Definition 1 Let $A(\mathbf{P})$ be a formula and $\Phi(\mathbf{P})$ be a tuple of formulas which is broken into $\Phi^{1}(\mathbf{P}), \Phi^{2}(\mathbf{P}), \ldots, \Phi^{k}(\mathbf{P})$ where $\mathbf{P}$ is a tuple of predicates used in these formulas.

The syntactic definition of prioritized circumscription is as follows:

$$
A(\mathbf{P}) \wedge \neg \exists \mathbf{p}(A(\mathbf{p}) \wedge \Phi(\mathbf{p}) \succ \Phi(\mathbf{P})),
$$

where:

1. $\mathrm{p}$ is a tuple of predicate variables each of which has the same arity as the corresponding predicate constant in $\mathbf{P}$,

2. $A(\mathbf{p})(\Phi(\mathbf{p}))$ is a formula obtained by replacing every occurrence in $A(\Phi$, respectively) of a predicate constant in $\mathbf{P}$ by the corresponding predicate variable in $\mathbf{p}$. 
According to the result of (Litischily, 1985). we give a model theoretic definition of the above formula (1) as follows.

Definition 2 We define an order $\geq$ oner logical interprelations as follows:

where

$$
M^{\prime} \geq M
$$

1. $M^{\prime}$ and $M$ have the same domain.

2. centy constant and function symbol has the. same interpretation in $M^{\prime}$ and $M$.

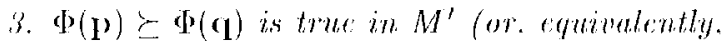
in $M)$ for $M^{\prime}[\mathbf{P}]$ a.s $\mathbf{P}$ and $M[\mathbf{P}]$ ass of where $M^{\prime}[\mathbf{P}](M[\mathbf{P}])$ is a tuple of the extensions for $M^{\prime}(M$. respectinely) of predicates in $\mathbf{P}$

In the above oreler, a wreater interpretation is tuore preferable. 'The above order intuitively neans that logical interpretations which maxjmally satisfy a subset of $\Phi^{1}$ are preferable, and if there are interpretations which satisfy the same formulas in $\Phi^{1}$. then interpredations which maximally satisfy a subset of $\Phi^{2}$ are preferable, and... and if there are interpretations which satisfy the same formulas in $\Phi^{k-1}$, then interpretationss which naxinally satisfy a subset of $\Phi^{2}$ are preforable.

Let. $A$ be a formula. We say that a logical interpretation $M$ is the most preferable model in the class of models of $A$ w.r.t. $\geq$ if thene is no model $M^{\prime}$ of $A$ in the class such that $M^{\prime} \geq M$ and not $M \geq M^{\prime}$

According to the result of (Lifichliti, 1985), wo lave the following correspondence between synLactic defintion and semantic defintion.

Theorem 1 A logical interpetation $M$ is a model of (1) iff $M$ is the most preferuble moded w.r.t. $\geq$ in the class of models of $A$.

\section{Disambiguation by Prioritized Circumscription}

In order to nse priontized circumseription for a disandbigtation lask, we make the following correspondence between formulas in the definition of prioritized circumscription and information in natural language. In the syntactic elelintion of prioritized circtunseription in Section 2, we correspond $A$ with information about given sentences and backgronud knowledge which is always true in any situation. And, we regard $\Phi$ as a tinple of preference rules. Note that preference mules are put into liverarehy according to sterength of preference rules. 'Then, the most preferatse models cor'respond with the most preferable realings since ach model satislies stronger preference rules as numch as possible and therefore, the syntactic defintition becomes a specification of the preferables readings by Theorem 1.

In the subsecpuent subsections, we tirstily tix an experinental logical representation of sentences, backeround knowledge and preferences. Then, we treat the exanple in Section 1 by the logical representiation.

\subsection{Logical Representation of Sentences and Background Knowledge}

We 11 se an adaptation of Kowalski's event calculus (Kowalski and Sergot, 1986). However, the idea of disambignation in this paper does not depencl on a particular representation. We assume that each sentence expresses an event. For exannple, a sentence "John gave the telescope to the man" is represented as the following formula.

act $(k$, Crive $) \wedge \operatorname{actor}(\omega, J o h n) \wedge$

objecl( $F, T$ descope $) \wedge$ recipient $(F, M a n)$

A complex sentience is supposed to be decomprosed intio a set of simple sentences which is translated into the above representation. Ambiguities arce expressed by disjunctions. For example, the sentence "John saw a man with a telescope" is expressed as follows.

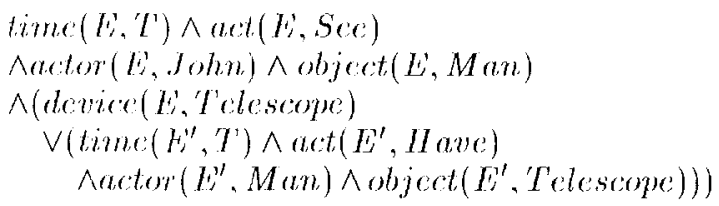

The last conjunct expresses ambiguity in the phrabe "with a telescope" (used as a device or carried by the man).

In adilition to the semantic representation, we also use syntactical information from a parser so that grammatical preference rules can be expressed. For example, we show some of the granmatical information of the sentience "John gave lihe telescope to the man" as follows. (We assunte that sentence $11+m b$ ber is 1 ).

$$
\begin{aligned}
& \text { subj(1, fohn } \wedge \text { verb(1, Gine }) \\
& \text { Adircet_obj(1, Telescope) } \\
& \text { Aindirect_obj(1, Man }) \\
& \text { Ain_the_sentence(1,John) }
\end{aligned}
$$

By using these basic predicates, we can represent backgronud knowledge which are always valid. For exanple, backgronnd knowledge "If $a_{1}$ lass at time $i_{\text {, and }} a_{1}$ is not equal to $a_{2}$, liten $a_{2}$ does not have o at litue " can be expressed in the following formula ${ }^{2}$.

$$
\begin{aligned}
& \forall e \forall i \forall a_{1} \forall a_{2} \forall \circ \forall c_{1}( \\
& \text { (time }(e, i) \wedge \text { act }(e, H a v e) \wedge \text { actor }\left(e, a_{1}\right) \\
& \left.\wedge \text { object }(c, o) \wedge \neg e q\left(a_{1}, a_{2}\right)\right) \supset \\
& \left(\left(\text { lime }\left(e_{1}, i\right) \wedge \text { act }\left(e_{1}, \text { Have }\right)\right.\right. \\
& \text { Aaclor } \left.\left.\left.\left(c_{1}, a_{2}\right)\right) \supset \text { ablject }\left(c_{1},()\right)\right)\right)
\end{aligned}
$$

\subsection{Logical Representation of Preferences}

We represent a preference rule as a formula in $\Phi$ in 1.he syntactic definition of prioritized circumscription and handle a priority among preferences by

${ }^{5}$ Wo ignore joint ownership for simplicity. If we world like to consider the possibility, we can represent the fornula as the strongest preference. 
putting stronger preferences into a stronger hierarchy of preferences.

For example, consider the following two grammatical preferences.

1. If "He" appears in a sentence as the subject and the subject in the previous sentence is male, then it is preferable that "He" refers to the previous subject.

2. If "He" appears in a sentence as the subject, and someone in the previous sentence is male, then it is preferable that " $H e$ " refers to the one in the previous sentence.

Suppose that the former is stronger than the latter. This priority of the preferences means that the formula:

$$
(i s a(a, \text { Male }) \wedge \operatorname{subj}(i, a)
$$$$
\text { Ainthe_sentence }(i+1, H e)) \supset e q(a, H e
$$

should be satisfied as much as possible for every $a$ and $i$, and if it is maximally satisfied then the following formtila:

$$
\text { (isa }(a, \text { Male }) \wedge i n_{\text {_t the_sentence }}(i, a)
$$$$
\wedge i n \text { the_sentence }(i+1, H e)) \supset e q(a, H e)
$$

should be satisfied as much as possible for every $a$ and $i$.

We can represent semantic preferences as well. For example, a preference "If $a_{1}$ sees $a_{2}$, then $a_{2}$ and $a_{1}$ are not equal" means that the following expression should be satisfied as much as possible for every $e, a_{1}$ and $a_{2}$ :

$$
\begin{aligned}
& \left(\operatorname{act}(e, S e e) \wedge \operatorname{actor}\left(e, a_{1}\right) \wedge \operatorname{object}\left(e, a_{2}\right)\right) \supset \\
& \quad \neg \text { eq }\left(a_{2}, a_{1}\right)
\end{aligned}
$$

Note that the above is a preference rule because there is a possibility of reflexive use of "see".

\subsection{Example}

Now, we are ready to treat disambiguation of the sentences used in Section 1 by prioritized circumscription.

We consider the following background knowledge which is always true. We denote the conjunctions of the following axioms as $A_{0}(\mathbf{P})$ where

$\mathbf{P} \stackrel{\text { def }}{=}\langle e q, i s$, time, act, actor, object, recipient, device, subj, in_the_sentence $\rangle$.

1. If $a_{1}$ is equal to $a_{2}$ then $a_{2}$ is equal to $a_{1}$. $\forall a_{1} \forall a_{2}\left(e q\left(a_{1}, a_{2}\right) \supset e q\left(a_{2}, a_{1}\right)\right)$

2. If $a_{1}$ and $a_{2}$ are equal and $a_{2}$ and $a_{3}$ are equal, then $a_{1}$ and $a_{3}$ are equal.

$$
\begin{aligned}
& \forall a_{1} \forall a_{2} \forall a_{3}\left(\left(e q\left(a_{1}, a_{2}\right) \wedge e q\left(a_{2}, a_{3}\right)\right) \supset\right. \\
& \left.\quad e q\left(a_{1}, a_{3}\right)\right)
\end{aligned}
$$

3. If $a_{1}$ is equal to $a_{2}$, then $a_{2}$ is an actor of $a_{1}$ 's action, troo.

$$
\begin{aligned}
& \forall e \forall a_{1} \forall a_{2}\left(\left(e q\left(a_{1}, a_{2}\right) \wedge \operatorname{actor}\left(e, a_{1}\right)\right) \supset\right. \\
& \left.\quad \text { actor }\left(e, a_{2}\right)\right)
\end{aligned}
$$

4. If $a$ use $o$ as a device at time $i$ then $a$ has $o$ at time $i$.

$$
\begin{aligned}
& \forall e \forall i \forall a \forall o((t i m e(e, i) \wedge \operatorname{actor}(e, a) \\
& \wedge \text { device }(e, o)) \supset \\
& \exists e_{1}\left(\operatorname{time}\left(e_{1}, i\right) \wedge \operatorname{act}\left(e_{1}, H a v e\right)\right. \\
& \left.\left.\wedge \operatorname{actor}\left(e_{1}, a\right) \wedge \operatorname{object}\left(e_{1}, o\right)\right)\right)
\end{aligned}
$$

5. If $a_{1}$ has $o$ at time $i$, and $a_{1}$ is not equal to $a_{2}$, then $a_{2}$ does not have $o$ at time $i$.

This is same as (2).

We consider the following preferences.

1. If $a_{1}$ sees $a_{2}$, then $a_{1}$ and $a_{2}$ are not equal. $\Phi_{1}^{1}\left(\mathbf{P}, e, a_{1}, a_{2}\right)=(5)$

2. If $a$ is male and $a$ is the subject of $i$-th sentence and "He" is in the next sentence, then $a$ is equal to "He".

$$
\Phi_{1}^{2}(\mathbf{P}, e, a, i)=(3)
$$

3. If $a$ is male and $a$ is in $i$-th sentence and "He" is in the next sentence, then $a$ is equal to "He".

$$
\Phi_{1}^{3}(\mathbf{P}, a, i)=(4)
$$

4. If someone gives $o$ to $a$ at time $i$, then $a$ has $o$ at time $i+1$. This expresses inertia of ownership.

$$
\begin{aligned}
& \Phi_{1}^{4}(\mathbf{P}, e, a, o, i)= \\
& (\operatorname{act}(e, \text { Give }) \wedge \text { object }(e, o) \\
& \wedge \text { recipient }(e, a) \wedge \text { time }(e, i)) \supset \\
& \quad \exists e_{1}\left(\operatorname{act}\left(e_{1}, H a v e\right) \wedge \operatorname{actor}\left(e_{1}, a\right)\right. \\
& \left.\quad \wedge \text { object }\left(e_{1}, o\right) \wedge \operatorname{time}\left(e_{1}, i+1\right)\right)
\end{aligned}
$$

5 . If $a$ buys $o$ at time $i$, then $a$ has $o$ at time $i+2$. This preference of another incrtia of ownership is weaker than the former preference because time interval is longer than the former preference.

$$
\begin{aligned}
& \Phi_{1}^{5}(\mathbf{P}, e, a, o, i)= \\
& (\operatorname{act}(e, B u y) \wedge \operatorname{actor}(e, a) \\
& \wedge o b j e c t(e, o) \wedge \text { time }(e, i)) \supset \\
& \exists e_{1}\left(\operatorname{act}\left(e_{1}, \text { Have }\right) \wedge \operatorname{actor}\left(e_{1}, a\right)\right. \\
& \left.\quad \wedge \operatorname{sobject}\left(e_{1}, o\right) \wedge \operatorname{time}\left(e_{1}, i+2\right)\right)
\end{aligned}
$$

We assume that $\Phi_{1}^{1}$ is a formula which should be satisfied in the first place, $\Phi_{1}^{2}$ in the second place, $\Phi_{1}^{3}$ in the third place, $\Phi_{1}^{4}$ in the fourth place and $\Phi_{1}^{5}$ in the fifth place.

Example 1 We consider the following sentences. John just saw a man with a telescope. He bought the telescope yesterday.

A logical representation of the above sentences is as follows and we denote it as $A_{1}(\mathbf{P})$.

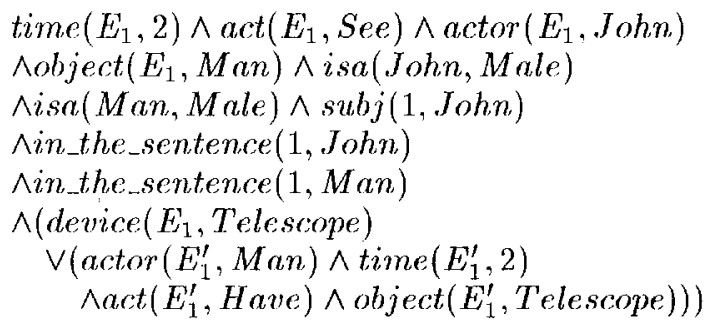




$$
\begin{aligned}
& \wedge \operatorname{time}\left(E_{2}, 0\right) \wedge \text { act }\left(E_{2}, B u y\right) \\
& \wedge a c t o r\left(E_{2}, H e\right) \wedge \text { object }\left(E_{2}, \text { Telescope }\right) \\
& \wedge i n \text { the sentence }(2, H e)
\end{aligned}
$$

Note that we represent, "just" as time 2 and "yesterday" as time 0.

In the syntactic definition of the most preferable reacling (1), we let $A(\mathbf{P})$ be $A_{1}(\mathbf{P}) \wedge A_{1}(\mathbf{P})$ and $k$ be 5 .

We show an intuitive explanation of inference of gettiug the nost preterable reading as follows. From the preference 2, "The" preferably refers to John. Note that, although the preference 3 seens to be applicable, it is not actially used since the stronger preference 2 overrides the preference 3 .

Then, from the preference 5, Joln hat the telescope al time 2. From the preference 1, John is not equal to the man. Then, the man cannot lave the telescope at time 2 from the backgronnd knowledge 5 and therefore, the telescope was used as a device from the disjunction in $A_{1}(\mathbf{P})$. We can actually prove that device ( $s_{1}$, telescope) is true in the most preferable readings.

Example 2 Suppose we add the following sentenef to the previous sentences.

But, he gave the telescope to the man this morning.

A logical representation related to this sentence is as follows. We denote the formula as $\Lambda_{2}(\mathbf{P})$.

time $\left(E_{3}, 1\right) \wedge \operatorname{act}\left(E_{3}\right.$, Give $) \wedge \operatorname{actor}\left(E_{3}, H c\right)$

Aobject $\left(H_{3}\right.$, Telescope $) \wedge$ recipient $\left(E_{3}, M a n\right)$

Note that we represent "this morning" as time 1.

In this case, we let $A(\mathbf{P})$ be $A_{0}(\mathbf{P}) \wedge A_{1}(\mathbf{P}) \wedge$ $A_{2}(\mathbf{P})$ in the syntactic definition. Then, reading of "with a telescope" is changed. From the preference 4 , the man should have had the telescopes at tine 2. If the telescope were used as a device at lime 2, John would also have the telescope at the same time according to backgromul knowlelge 4 and it, contradicts backgromend knowledge 5 . Then, the weaker preference 5 is retracted to avoid contradiction and the stronger preference 4 is survived. 'Therofore, in the most preferable reading, the man had the telescope at time 2 .

\section{HCLP language}

Now, wo discuss an inplenentation of prioritized circumscription by IICTP. Firstly, we briefly review a hierarchical constraint logic programming(HCLP') language. We follow the definition of (Boruing of al., 1989).

An HCLP program consists of rules of the form:

$$
h:-b_{1}, \ldots, b_{n}
$$

where $h$ is a predicate and each of $b_{1}, \ldots, b_{n}$ is a predicate or a constraint or a labeled constraint. A labeled constrainti is of the form:

label $C$ where $C$ is a constraint in specific domain and label is a label which expresses strength of the constraint $C$.

The operational semantics for HCLP is similar to CLP except manipulating a constraint hierarchy. In HCLP, we accumulate labeled constraints to form a constraint hicrarchy by each label while executing CLP until CLP solves all goals and gives a reduced required constraints. Then, we solve constraint hierarchy with required constraints.

'Lo solve constraint hierarchy, we firstly find a maximal subset of constraints for the strongest; level which is consistent with the required constraints. 'Then, we try to find a maximal subset of constraints in the second strongest level with respect to the union of the required constraints and the maximal consistent subset for the strongest level,.., and so on until a maximal consistient subset of constraints in the $k$-th strongest level is arded. Then, an assignment which satisfies the final set of constraints is called a solution.

Iet $\theta$ and $\sigma$ be assignments and $C_{\theta}^{1}\left(\right.$ and $\left.C_{\sigma}^{1^{1}}\right)$ be a set of constraints in the strongest level of the hierarchy satisfied by $\theta\left(\right.$ and $\sigma$ ), and $C_{\theta}^{2}$ (and $C_{\sigma}^{2}$ ) be a set of constraints in the second strongest level of the hierardiy satisfied by $\theta($ and $\sigma) \ldots$, and $C_{\theta}^{k_{i}}\left(\right.$ and $\left.C_{\sigma}^{k}\right)$ be a set of constraints in the $k$-th strongest level of the hicrarchy satisfied by $\theta$ (and $\sigma)$.

$\theta$ is locally-predicate-better (Borning et al., 1989) than $\sigma$ w.r.t. the constraint hierarchy if there exists $i(1 \leq i \leq k)$ such that for every $j(1 \leq j \leq i \cdots 1), C_{\sigma r}^{j}=C_{\theta}^{j}$ and $C_{\sigma}^{i} \subset C_{\theta}^{i}$,

We can prove that if $\theta$ is a solution, then there is no assignment, $\sigma$ which satisfies the required constraints and is bocally-predicate-better than $\theta$.

Note that the definition of locally-predicatebetter comparator is similar to the definition of the order over logical interpretation in the prioritized circumscription. The difference is that locally-predicate-better comparator considers assignments for variables in constraints in HCLP whereas the order over logical interpretation considers assignments of truth-value for formulas in prioritized circumscription.

\section{Implementation by HCLP language}

In order to nse HCLP' language for implenentiation of prioritized circumscription, we need to change formulas in prioritized circunscription into constraints in HCLP. It is done as follows. We introduce a domain closure axiom so that we only consider relevant constants used in the given sentences. Then, we instantiate universal-quantified variables in background knowledge and free variables in preferences with the relevant constints and introduce Skolem functions for existentialquantified variables. 
For example, we lave the following formula by instantiating preference 4 in Section 3.3 with $F_{3}$ for $e$ and the man for $a$ and the telescope for $o$ and 1 for $i$ and introducing a Skolem function $f$ :

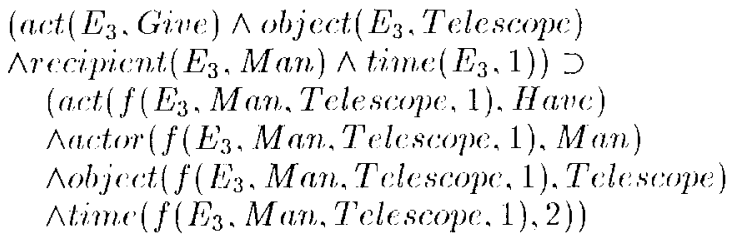

By this translation, every formula becomes ground and we regard a different gromed atom as a different propositional symbol. Then, every formula in prioritized circunscription can be regarded as a boolean constraint in HCLP. We translate all formulas in the syntactic definition of the backgronnd knowledge and the sentences in Exanples 1 and 2 into boolean constraituts in onr IICLI' langnage (Satoh, 1990). 'Then, from the two sentences in Fxample 1, onr IICLP language gives the following result as a part of a solution:

$$
\begin{aligned}
& \text { time }\left(E_{1}, 2\right)=\text { true } \\
& \text { actor }\left(E_{1}, J \text { ohn }\right)=\text { true } \\
& \text { object }\left(E_{1}, M a n\right)=\text { true } \\
& \text { act }\left(E_{1}, S e c\right)=\text { true } \\
& \text { demee }\left(E_{1}, \text { Telescope }\right)=\text { true }
\end{aligned}
$$

which means that the telescope is ned as a device.

And, onr HCLP language gives tho following result for the sentences in Example 2:

$$
\begin{aligned}
& \text { time }\left(l_{1}, 2\right)=\text { true } \\
& \operatorname{ardor}\left(E_{1}, J_{0 h n}\right)=\text { true } \\
& \operatorname{object}\left(F_{1}, M a n\right)=\text { true } \\
& \text { act }\left(L_{1}, \text { See }\right)=\text { truce } \\
& \text { derice }\left(E_{1}, T e l e s c o p e\right)=\text { false } \\
& \operatorname{actor}\left(b_{1}^{\prime}, \operatorname{Man}\right)=\text { true } \\
& \text { time }\left(E_{1}^{\prime \prime}, 2\right)=\text { true } \\
& \operatorname{act}\left(E_{1}^{\prime}, \text { Have }\right)=\text { true } \\
& \text { object }\left(E_{1}^{\prime \prime} \text { Trlescope }\right)=\text { true }
\end{aligned}
$$

which means that the man has the telescope (and it is not tued as a device).

\section{Conclusion}

We believe that the following are contributions of this paler.

1. Wo examine a feasibility of prioritized circumscription for specifying the nost preferable reading by considering a disanbiguation task in the concrete examples and show that we can represent the task quite naturally.

2. We disenss an implementation of clisambignation within an HCLP language by showing a correspondence between a priority over preference rules in prioritized circonnscription and a constraint hierarchy in HCIP.

As a future research, we need the following.

1. We would like to examine a computational complexity of dismuliguation by HCIP.
2. It is better to learn preferences antomatically in stead of specifying preferences by nser. One approach for learning is to build an interactive system such that the system shows to a user a set of possible readings for given sentences and the nser gives an order over possible reaclings. Then, the system would be able to learn preferences by generalizing the order.

\section{References}

Borning, A. Maher, M., Martindale, A., and Wilson, M. 1989. Constiraint Hicrarchies and Logic Programming. In Procedings of the Intermational Conference on Logic Programming, pages 149164.

Charniak, E., and Goldman, R. 1989. A Semantics for Probabilistic Quantificr-Free FirstOrder Languages, with Particular Application to Story Understanding. In Proceedings of IJCAI-89, pages 10741079.

Hobbs, J. R. 1990. Two Principles of Parse Preference In Proceedings of COLING-90, Vol. 3 , pages 162167.

Hobbs, J. R., Stickel, M., Martin, P. and Edwards, D. 1993. Interpretation as Abduction. Artificial Intelligence, Vol. 63, pages 69142.

Kancyama, M., 1994. Indefeasible Semantics and Defeasible Pragmatics. SRI Technical Note. 544, SRI, 30 pages.

Kowalski, R. and Sergot, M. 1986. A Iogic-based Calculus of Events. New Generation Computing. Vol. 4, pages 6795.

Lascarides. A. and Asher, N. 1993. Temporal Interpretation, Disconrse Relations, and Commonsense Fntailment. Linguistics and Philosophy, Vol. 16, pages 437493.

Lifschitz, V. 1985. Computing Circunnscription. In Proceedings of IJCAI-85, pages $121 \quad 127$.

McCarthy, J. 1986. Applications of Ciscumscription to Formalizing Commonsense Knowledge. Artificial Intelligence, Vol. 28, pages 89116.

Quantz, J. J., 1993. Interpretation as Exception Minimization. In Procedings of I. CA $1-93$. pages 1310 1315 .

Satol, K., 3991. A Jogical Fonndation of Preference-based Disambiguation. ICOT-Th638, ICO'T, 15 pages.

Satoh, K and Aiba, A. 1990. The Hierarchical Constiaint Logic: Language: CHAL, ICOT'T'R-592, ICO'T, 18 pages.

Wilks, Y. 1975. A Preferential, Pattern-Seeking, Semantices for Natural Language Inference $A r$ tificial Intelligence, Vol 6, pages 5374.

Zernik, U. and Brown, A. 1988. Defantt Reasoning in Natinal Language Processing. In Proceedings of COLING-88, pages. 801. 805. 